\title{
DIAMAGNETIC CAVITIES IN THE INTERSTELLAR MEDIUM
}

\author{
GALEN GISLER \\ Space Plasma Physics, ESS-8, Mail Stop D-438 \\ Los Alamos National Laboratory \\ Los Alamos, New Mexico \\ USA 87545
}

\begin{abstract}
Violent events such as supernova explosions or gradual processes such as mass loss from hot supergiant stars produce diamagnetic cavities: the interstellar magnetic field, frozen into ambient plasma, is excluded from a region around the source of expanding injected plasma. Particle-in-cell simulations illustrate the formation of such diamagnetic cavities in both the steady and impulsive cases. Plasma loss along the field means that the cavity always tends toward a cylindrical or barrel shape, even for very weak magnetic fields. Ion acceleration is expected out the ends of the barrel for all parameters of injection and initial field.
\end{abstract}

Charged particles at the leading edge of a hot plasma expanding into an ambient magnetic field gyrate on encountering the field. This gyration produces a layer of current that reduces the magnetic field to its interior, hence the expansion is diamagnetic. This current layer moves out with the expanding plasma, and the resulting magnetic field gradient acts to resist the radial expansion of the plasma. The expansion in the direction along the field, on the other hand, is almost free. The analysis of Gisler and Lemons (1989) and the particle-in-cell simulations of Gisler (1989) show that these kinematical effects result in the plasma adopting a geometry that conforms to the ambient field, even for very high ratios of kinetic to field energy density. Hence field-aligned barrel shapes or clam shells should be common among SNR independent of source considerations.

In the simulations of Gisler (1989), and in additional simulations performed for this meeting (to be published in greater depth elsewhere) ion acceleration is observed along the axis of the ambient field, for all geometries, ficld strengths and injection time scales. Some ions achieve energies many times their initial thermal energies; indeed their velocities approach the electron thermal velocities. These accelerated ions may contribute importantly to the galactic cosmic ray seed population.

Heating of both ions and electrons occurs at the sharp radial edge of the diamagnetic cavity, especially at and subsequent to maximum expansion, when that edge is strongly decelerated. During the recompression, this is effectively first-order Fermi acceleration of trapped particles. Because plasma has been lost along the field, energy expended in the radial expansion is returned to a smaller mass of plasma during the recompression phase.

\section{References}

Gisler, Galen (1989) "Axisymmetric particle-in-cell simulations of diamagnetic-cavity formation in vacuum", IEEE Transactions on Plasma Science, 17, 210.

Gisler, Galen, and Lemons, Don S. (1989) "Dynamics of a plasma expanding into a uniform magnetic field", Journal of Geophysical Research, in press.

R. Beck et al. (eds.), Galactic and Intergalactic Magnetic Fields, 168.

(C) 1990 IAU. Printed in the Netherlands. 